\title{
Brownian motion in thin sheets of viscous fluid
}

\author{
By P. G. SAFFMAN \\ Applied Mathematics, California Institute of Technology, Pasadena
}

(Received 2 September 1975)

The drag on a cylindrical particle moving in a thin sheet of viscous fluid is calculated. It is supposed that the sheet is embedded in fluid of much lower viscosity. A finite steady drag is obtained, which depends logarithmically on the ratio of the viscosities. The Einstein relation is used to determine the diffusion coefficient for Brownian motion of the particle, with application to the movement of molecules in biological membranes. In addition, the Brownian motion is calculated using the Langevin equation, and a logarithmically time-dependent diffusivity is obtained for the case when the embedding fluid has zero viscosity.

\section{Introduction}

We consider a plane infinite sheet of viscous fluid of thickness $h$ lying between the surfaces $z=0$ and $z=-h$. A solid particle lies in the sheet. For definiteness, suppose that it is a circular cylinder of height $h$ and radius $a$, with generators perpendicular to the plane of the sheet and ends in the surfaces. The problem is to calculate the drag and torque on the cylinder when it moves with a variable velocity $U(t)$ and rotates about its axis with angular velocity $\Omega(t)$ under conditions such that the Reynolds number is small and the convective acceleration terms $\mathbf{u} . \nabla \mathbf{u}$ in the equations of motion may be neglected.

This problem is relevant to the diffusion of protein and lipid molecules in biological membranes (Saffman \& Delbrück 1975). Experimental techniques have recently been developed which allow the diffusion, both transitional and angular, to be measured (see Edidin 1974). The experimental evidence demonstrates that the membrane is fluid and the diffusion is due to Brownian motion. The theory of Brownian motion of a particle in a fluid (see Einstein 1956) relates the mean-square displacement or rotation to a diffusion coefficient $D$ given by

$$
D=k_{B} T b,
$$

where $b$ is the (constant) mobility of the particle in the fluid, $k_{B}$ is Boltzmann's constant and $T$ is the absolute temperature. (See also Landau \& Lifshitz 1959, $\S 59$.) The mobility is defined as the steady velocity (angular velocity) produced by a steady unit force (torque), as given by solutions of the creeping-flow equations.

If the particle were a sphere of radius $a$ in a fluid of viscosity $\mu$, it is well known that

$$
b_{T}=(6 \pi \mu a)^{-1}, \quad b_{R 2}=\left(8 \pi \mu a^{3}\right)^{-1},
$$


where the suffixes $T$ and $R$ refer to translation and rotation, respectively. The combination of (1.1) and (1.2) is referred to as the Stokes-Einstein relation. These formulae have been applied to the diffusion of large molecules in biological membranes, but this procedure is not altogether sound as the model is inadequate. A better approach is to calculate the mobility for a more realistic model, such as that described in the opening paragraph, and this is the purpose of the present paper.

The membrane usually separates two fluids (aqueous phases) of much smaller viscosity. In the first instance, we can therefore neglect the tangential stresses on the surfaces of the sheet, which models the membrane. The motion in the sheet is then two-dimensional, with no variation of flow properties across the sheet. Thus the drag and torque are those on a length $h$ of a cylinder moving through a viscous fluid.

The calculation of the torque is trivial. With polar co-ordinates $(r, \theta)$, the tangential velocity $u_{\theta}$ for steady rotation of a cylinder of radius $a$ with angular velocity $\Omega$ is $\Omega a^{2} / r$. The tangential stress is $2 \mu \Omega$ and hence the torque is $4 \pi \mu \Omega a^{2} h$. Thus

$$
b_{R}=\left(4 \pi \mu a^{2} h\right)^{-1} .
$$

However, the translational mobility is a different matter, because this mobility is infinite. A steady force per unit length applied to an infinitely long cylinder gives an infinite velocity, according to the creeping-flow equations (the Stokes paradox, Lamb 1932, §343). To obtain a finite mobility so that the Einstein relation (1.1) can be employed, we can either introduce the convective acceleration terms (i.e. use the Oseen drag), or realize that the membrane is of finite size and obtain a mobility which varies inversely with the logarithm of the sheet radius, or take account of the viscosity of the aqueous fluid outside the sheet. The first approach is not viable because it is not enough for the mobility to be finite: it must be constant, independent of the velocity, for the Einstein formula. The second approach is simple; the result will be given below in equation (3.13). The third appears, on examination of the results and insertion of typical values for the parameters, to be most relevant. Accordingly, we shall discuss in $\S \S 2$ and 3 the drag on a cylindrical particle moving through a sheet of viscous fluid which separates two other fluids of much smaller viscosity. A finite drag and (constant) mobility are obtained, and hence a coefficient of translational diffusion. It will be seen that the diffusion coefficient depends logarithmically on the ratio of the viscosities.

An alternative procedure is to employ the Langevin equation (Wax 1954, p. 22) and calculate the diffusion of a particle directly in terms of the relation between drag and past acceleration for a cylinder in an infinite viscous fluid. In this way an effective diffusivity is calculated which is a logarithmic function of the time. This result is not relevant to the biological application for the usual range of the parameters, but the argument is of some mathematical and physical interest, particularly because of recent developments in non-equilibrium thermodynamics, and will be described in $\$ \$ 4$ and 5 below. 


\section{Drag on a particle in a sheet}

A viscous fluid, of viscosity $\mu$, occupies the region $-h<z<0$, and contains a cylindrical particle of radius $a$ and height $h$, which moves with constant velocity $U$ parallel to the $x$ axis. Take polar co-ordinates in the plane of the sheet, with origin lying instantaneously on the axis of the particle.

The regions $z>0$ and $z<-h$ are occupied by another fluid of viscosity $\mu^{\prime}$. We are particularly interested in the case $\mu^{\prime} \ll \mu$. Both fluids are incompressible.

In $z>0$, the equations of motion are

$$
0=-\nabla p^{\prime}+\mu^{\prime} \nabla^{2} \mathbf{u}^{\prime}, \quad \operatorname{div} \mathbf{u}^{\prime}=0,
$$

where $\mathbf{u}^{\prime}=\left(u_{r}^{\prime}, u_{\theta}^{\prime}, u_{z}^{\prime}\right)$. On the surface of the particle $z=0, r<a$, we have the boundary condition

$$
u_{r}^{\prime}=U \cos \theta, \quad u_{\theta}^{\prime}=-U \sin \theta \quad(r<a, \quad z=0) .
$$

The surfaces of the sheet are supposed rigid, so that

$$
u_{z}^{\prime}=0 \text { for } r \geqslant 0, \quad z=0 .
$$

It may be noted that the solution we shall find (in §3) for $\mu^{\prime} \ll \mu$ remains valid if the particle sticks out of the sheet. The boundary condition (2.2) serves the purpose of making the problem definite, and exposing the symmetries of the flow field.

By taking Hankel transforms, we find that the general solution of (2.1) and (2.3), with the angular symmetry of (2.2), is

$$
\begin{aligned}
& u_{r}^{\prime}=\cos \theta \int_{0}^{\infty}\left[\frac{1}{2} B J_{2}(k r)+\frac{1}{2} E J_{0}(k r)+\frac{1}{4}(E-B) k z\left\{J_{2}(k r)-J_{0}(k r)\right\}\right] e^{-k z} d k, \\
& u_{\theta}^{\prime}=\sin \theta \int_{0}^{\infty}\left[\frac{1}{2} B J_{2}(k r)-\frac{1}{2} E J_{0}(k r)+\frac{1}{4}(E-B) k z\left\{J_{2}(k r)+J_{0}(k r)\right\}\right] e^{-k z} d k, \\
& u_{z}^{\prime}=\frac{1}{2} \cos \theta \int_{0}^{\infty}(E-B) k z J_{1}(k r) e^{-k z} d k, \\
& p^{\prime}=\mu^{\prime} \cos \theta \int_{0}^{\infty} k(E-B) J_{1}(k r) e^{-k z} d k .
\end{aligned}
$$

Here, $B$ and $E$ are arbitrary functions of $k$. The fluid in $z>0$ exerts a surface stress $\sigma=\left(\sigma_{r}, \sigma_{\theta}\right)$ on the sheet, where

$$
\sigma_{r}=\left.\mu^{\prime} \frac{\partial u_{r}^{\prime}}{\partial z}\right|_{z=0}, \quad \sigma_{\theta}=\left.\mu^{\prime} \frac{\partial u_{\theta}^{\prime}}{\partial z}\right|_{z=0} .
$$

An equal stress is exerted by the fluid in $z<-h$.

We turn now to the equation of motion of the fluid in the sheet. Because of the molecular structure of the membrane, there can be no variation of velocity across it, and it should be modelled by a non-isotropic fluid, such that motions in the plane of the sheet give viscous stresses described by a Newtonian coefficient 
of viscosity $\mu$, but $\partial / \partial z=0$. Then the velocity $\mathbf{u}=\left(u_{r}, u_{\theta}, 0\right)$ in the sheet satisfies, for $r>a$,

$$
0=-\nabla p+\mu \nabla^{2} \mathbf{u}+2 \boldsymbol{\sigma} / h, \quad \operatorname{div} \mathbf{u}=0 .
$$

The last term in the momentum equation describes the effect of the drag $2 \sigma$ distributed through the sheet of width $h$.

In addition, we assume that the fluid does not slip at the surface of the sheet, i.e.

$$
u_{r}(r, \theta)=u_{r}^{\prime}(r, \theta, 0), \quad u_{\theta}(r, \theta)=u_{\theta}^{\prime}(r, \theta, 0) .
$$

These equations hold for all $r$. Hence $(2.4 a, b)$, evaluated at $z=0$, give Hankel transforms for $u_{r}$ and $u_{\theta}$. Then the incompressibility equation leads, after some algebra, to the relation

$$
B=E \text {. }
$$

Simplifying the expressions, we have

and

$$
\left.\begin{array}{l}
u_{r}=\frac{1}{2} \cos \theta \int_{0}^{\infty} B\left(J_{2}+J_{0}\right) d k, \\
u_{\theta}=\frac{1}{2} \sin \theta \int_{0}^{\infty} B\left(J_{2}-J_{0}\right) d k,
\end{array}\right\}
$$

$$
\left.\begin{array}{l}
\sigma_{r}=-\frac{\mu^{\prime}}{2} \cos \theta \int_{0}^{\infty} k B\left(J_{2}+J_{0}\right) d k, \\
\sigma_{\theta}=-\frac{\mu^{\prime}}{2} \sin \theta \int_{0}^{\infty} k B\left(J_{2}-J_{0}\right) d k .
\end{array}\right\}
$$

The argument $k r$ of the Bessel functions is omitted for brevity.

We now substitute (2.9) and (2.10) into (2.6), eliminate $p$, and find

$$
\int_{0}^{\infty} B k^{3} J_{1}(k r)\left(1+\frac{2 \mu^{\prime}}{\mu k h}\right) d k=0 \quad \text { for } \quad r<a .
$$

The boundary condition (2.2) gives

$$
\int_{0}^{\infty} \frac{B}{k} J_{1}(k r) d k=U r \text { for } \quad r<a .
$$

These two equations constitute a dual integral equation for $B(k)$, which when found leads to the complete solution. Methods exist for treating such equations (Tranter 1966; Titchmarsh 1948), but they are not simple and here we shall make use of the fact that $\mu^{\prime} a / \mu h \ll 1$ for cases of interest, and solve directly by singular perturbation techniques.

\section{Approximate solution for $\mu^{\prime} \ll \mu$}

We calculate first the outer solution, obtained by supposing that the particle shrinks to a point and can be replaced by a point force $\mathrm{F}$ along $r=0,-h<z<0$, or a force density $\mathbf{F} \delta(r) / 2 \pi r h$, where $\mathbf{F}$ is the drag on the particle (and is in the opposite direction to $U$ ). Thus we replace (2.6) by

$$
0=-\nabla p+\mu \nabla^{2} \mathbf{u}+\frac{2 \boldsymbol{\sigma}}{h}+\frac{\mathbf{F} \delta(r)}{2 \pi r h}, \quad \operatorname{div} \mathbf{u}=0,
$$


which hold for all $r \geqslant 0$. The solution of (3.1) by Hankel transforms is straightforward and leads to

$$
B(k)=\frac{F}{2 \pi h} \frac{1}{\mu k+2 \mu^{\prime} / h},
$$

where $F=|\mathbf{F}|$. Therefore, after an elementary transformation, we find that the leading term in the outer expansion of the radial velocity (valid where $r / a \gg \mu / \mu^{\prime}$ ) is

$$
u_{r}=\frac{F \cos \theta}{4 \pi \mu h} \int_{0}^{\infty} \frac{J_{0}(\xi)+J_{2}(\xi)}{\xi+2 r h^{-1} \mu^{\prime} \mid \mu} d \xi
$$

We need the asymptotic form of this expression as $r \rightarrow 0$, i.e. the inner limit of the outer solution. By adding and subtracting the integral of the denominator from 0 to 1 , we obtain

$$
\begin{aligned}
\frac{4 \pi \mu h}{F \cos \theta} u_{r} & \sim-\log \left(\frac{2 r \mu^{\prime}}{h \mu}\right)+\int_{0}^{\infty} \frac{J_{2}(\xi)}{\xi} d \xi+\int_{0}^{1} \frac{J_{0}(\xi)-1}{\xi} d \xi+\int_{1}^{\infty} \frac{J_{0}(\xi)}{\xi} d \xi \\
= & -\log \left(2 r \mu^{\prime}(h \mu)+\frac{1}{2}-\gamma+\log 2\right.
\end{aligned}
$$

see Abramowitz \& Stegun (1965, equation 11.1.20).

To find the drag, we match to the leading term of the inner solution, i.e. the solution of the two-dimensional flow equations (2.6) with $\sigma=0$. The radial velocity field is obtained from solutions of the biharmonic equation and is

$$
u_{r}=\cos \theta\left[\frac{\alpha}{r^{2}}+\beta-\frac{F}{4 \pi \mu h} \log r\right]
$$

where $\alpha$ and $\beta$ are constants depending on the shape and size of the particle and the boundary conditions on its surface. It is a consequence of the equations of motion that the coefficient of $\log r$ is proportional to $F$, as can be demonstrated by evaluating the momentum flux through a large circle.

The matching requirement on (3.3) and (3.5), namely that (3.3) as $r \rightarrow 0$ agrees with (3.5) as $r \rightarrow \infty$, gives

$$
\beta=\frac{F}{4 \pi \mu h}\left[\log \frac{h \mu}{\mu^{\prime}}+\frac{1}{2}-\gamma\right]
$$

We now apply boundary conditions on the surface of the particle $r=0$. First, we assume a no-slip boundary condition,

$$
u_{r}=U \cos \theta, \quad u_{\theta}=-U \sin \theta \text { when } r=a .
$$

It follows that, since $\partial u_{\theta} / \partial \theta=-\partial\left(r u_{r}\right) / \partial r$,

$$
\frac{\alpha}{a^{2}}+\beta-\frac{F}{4 \pi \mu h} \log a=U, \quad \frac{\alpha}{a^{2}}-\beta+\frac{F}{4 \pi \mu h}+\frac{F}{4 \pi \mu h} \log a=-U . \quad(3.8 a, b)
$$

Hence, from (3.6) and (3.8),

$$
F=4 \pi \mu h U\left[\log \left(h \mu / a \mu^{\prime}\right)-\gamma\right]^{-1} .
$$

If, instead of a no-slip boundary condition, we assume zero tangential stress on $r=a$ (and it is believed by some workers that this might be a more appropriate 
boundary condition), it is easily seen that (3.8b) should be replaced by $\alpha=0$, and (3.9) is obtained with the term $+\frac{1}{2}$ added to the square bracket and a slightly smaller drag is found.

The translational mobility $b_{T}$ is $U / F$, and hence the Einstein relation (1.1) gives a coefficient of translational diffusion

$$
D_{T^{\prime}}=\frac{k_{B} T}{4 \pi \mu h}\left[\log \frac{h \mu}{a \mu^{\prime}}-\gamma\right] .
$$

A zero-tangential-stress boundary condition instead of the no-slip assumption adds $\frac{1}{2}$ inside the square bracket.

The coefficient of rotational diffusion follows from (1.3) and is

$$
D_{R}=k_{B} T / 4 \pi \mu h a^{2} \text {. }
$$

As discussed in the introduction, the Stokes paradox has led to a translational mobility which goes to infinity as the viscosity $\mu^{\prime}$ of the surrounding medium goes to zero. Reasonable estimates for biological membranes give $h \approx a$ and $\mu^{\prime} \mid \mu \approx 10^{-2}$. Thus $D_{T} / D_{R}$ should be about three times greater than values expected on the basis of the Stokes-Einstein formulae, valid for spherical particles.

Inspection of the analysis shows that it remains valid if the particle sticks out of the membrane, provided of course that $\mu^{\prime} \ll \mu$, because the outer flow field (3.3) and the inner one (3.5) are independent of particle penetration into the liquid embedding the sheet.

We can give now the result for the case when a finite mobility is obtained by giving the membrane a finite size rather than using the viscosity of the surrounding fluid. The radial velocity in the sheet is given by (3.5). The divergence as $r \rightarrow \infty$ is removed by restricting the flow to the interior of a circle of large radius $R$. In order that the velocity will be small when $r=R$, we must have

$$
\beta=(F / 4 \pi \mu h) \log R
$$

to replace (3.6). Then (3.9) is replaced by

$$
F=4 \pi \mu h U\left[\log (R / a)-\frac{1}{2}\right]^{-1} .
$$

If $R / a \gg h \mu / a \mu^{\prime}$, as can be expected, the finite-size effect is negligible. If $R / a \ll h \mu / a \mu^{\prime}$, then the mobility should be calculated from (3.13).

\section{Time-dependent diffusion in an infinite sheet bounded by inviscid fluid}

Although the case discussed in $\S 3$ appears to be that of practical value, it is of mathematical interest to consider the problem of diffusion in the limit when $\mu^{\prime} \rightarrow 0$ and $R \rightarrow \infty$. We now have an infinite mobility and the Einstein formula cannot be applied. The method now used is to study the Langevin equation for the particle in the sheet.

It is clearly sufficient to study one-dimensional motion. If $U(t)$ denotes the instantaneous velocity of the particle, the Langevin equation is

$$
M \frac{d U}{d t}+\int_{-\infty}^{t} S(t-\tau) U(\tau) d \tau=W(t)
$$


Here $M$ is the particle's mass, $S$ determines the macroscopic (viscous) drag in terms of past velocity, and $W(t)$ is a random force representing the random molecular bombardment. The key assumption embodied in the Langevin equation is that the force exerted by the medium on the particle can be broken up into two parts: a drag independent of the molecular structure of the medium and proportional to the velocity, and a rapidly fluctuating, stationary random force due to molecular vibrations and independent of the velocity. Simple justifications of the Langevin equation do not appear to be available. In addition, the random forcing term $W(t)$ must have properties consistent with the equipartition of energy. (This leads to the so-called fluctuation dissipation theorem.)

From (4.1), we calculate $U(t)$ as a stationary random function of time, with a velocity correlation

$$
R(\tau)=\langle U(t) U(t+\tau)\rangle,
$$

where angle brackets denote the average over the ensemble of molecular motions. The particle displacement in a time $t$ is

and it is well known that

$$
X(t)=\int_{0}^{t} U\left(t^{\prime}\right) d t^{\prime}
$$

$$
\left\langle X^{2}(t)\right\rangle=2 \int_{0}^{t}(t-\tau) R(\tau) d \tau \sim 2 t \int_{0}^{t} R(\tau) d \tau .
$$

When the last integral converges, this implies that the diffusion of the particle can be described by a diffusion coefficient

$$
\int_{0}^{\infty} R(\tau) d \tau
$$

A finite mobility ensures convergence of the integral [see (4.10)].

Let us suppose for the present that $S(t-\tau)$ is known. We shall discuss in $\S 5$ how it is to be calculated. Then $R(\tau)$ is calculated employing a form of the fluctuation dissipation theorem known as the relaxation function theorem (Kubo 1957). There is no difficulty in solving (4.1); taking Laplace transforms gives immediate answers. The problem is that the magnitude of $W(t)$ is unknown, and fixed by the equipartition of energy. We replace the problem described by (4.1) by that of the motion of a particle set instantaneously into motion with velocity $V_{0}$ and then relaxing to rest under viscous drag in the absence of the random forcing term. That is, we solve for $V(t)$, where

$$
\left.\begin{array}{c}
M \frac{d V}{d t}+\int_{0}^{t} S(t-\tau) V(\tau) d \tau=0, \quad V(0)=V_{0} \\
V(t)=0 \text { for } t<0 .
\end{array}\right\}
$$

$V_{0}$ is chosen such that the total kinetic energy, particle plus surrounding fluid, is $\frac{1}{2} k_{B} T$. The relaxation function theorem then states that

$$
R(\tau)=V_{0} V(\tau) \text {. }
$$

The argument for this theorem is obscure to the author. Alternative forms exist, but do not obviously lead to identical results for $R(\tau)$ although there is no 
doubt that all lead to the same asymptotic behaviour for $\left\langle X^{2}(t)\right\rangle$. However, when the mobility is infinite, there is at present no alternative.

To proceed, we note that (for incompressible fluid)

$$
\int_{0}^{t} S(t-\tau) V(\tau) d \tau=M^{\prime} \frac{d V}{d t}+\int_{0}^{t} G(t-\tau) V(\tau) d \tau
$$

where $G$ is integrable (in the class of generalized functions or distributions) and $M^{\prime}$ is the virtual mass of the particle. $M^{\prime} V_{0}$ is the impulse required to set the fluid instantaneously into motion and the initial kinetic energy is $\frac{1}{2} M^{\prime} V_{0}^{2}$. Laplace transforms are introduced, defined for a function $f(t)$ by

$$
\hat{f}(s)=\int_{0}^{\infty} e^{-s t} f(t) d t, \quad f(t)=\frac{1}{2 \pi i} \int_{c-i \infty}^{c+i \infty} \hat{f}(s) e^{s t} d s .
$$

It follows immediately from (4.5), and the requirement

that

$$
\frac{1}{2}\left(M+M^{\prime}\right) V_{0}^{2}=\frac{1}{2} k_{B} T,
$$

$$
\hat{R}(s)=\frac{k_{B} T}{M+M^{\prime}}\left[s+\frac{\hat{G}(s)}{M+M^{\prime}}\right]^{-1} .
$$

Note that the mobility is $1 / \hat{G}(0)$ and the diffusion coefficient is $\hat{R}(0)$, when these exist. Thus, in this case, (4.10) gives Einstein's relation.

For general $t$, application of the inverse Laplace transform to (4.4) and (4.10) gives

$$
\left\langle X^{2}(t)\right\rangle=\frac{2 k_{B} T}{M+M^{\prime}} \frac{1}{2 \pi i} \int_{c-i \infty}^{c+i \infty} \frac{e^{s t} d s}{s^{2}\left[s+\widehat{G}(s) /\left(M+M^{\prime}\right)\right]} .
$$

Thus the problem is reduced to the determination of $M^{\prime}$ and $\hat{G}(s)$, both being problems of classical hydrodynamics, and the evaluation of a contour integral.

For the purpose of illustration, consider the case of a spherical particle of radius $a$ in an unbounded viscous fluid of density $\rho$ and viscosity $\mu$. Then it is easily shown (see, for example, Landau \& Lifshitz, 1959, §24) that

$$
M^{\prime}=\frac{2}{3} \pi \rho a^{3}, \quad \tilde{G}(s)=6 \pi \mu a\left[1+\left(\rho a^{2} s \mid \mu\right)^{\frac{1}{2}}\right] .
$$

(Note that $G$ is to be interpreted as a generalized function.) Then, by standard techniques of asymptotic analysis,

$$
\left\langle X^{2}(t)\right\rangle \sim \frac{k_{B} T}{6 \pi \mu a}\left[2 t-4\left(\frac{\rho a^{2} t}{\pi \mu}\right)^{\frac{1}{2}}+\frac{\rho a^{2}}{\mu}-\frac{M+M^{\prime}}{6 \pi \mu a}+O\left(\frac{1}{t^{\frac{3}{2}}}\right)\right] .
$$

In the next section, we calculate $M^{\prime}$ and $G$ for a cylindrical particle in a sheet and find the mean-square displacement.

$\uparrow$ If the fluid is compressible, only the actual mass appears on the left-hand side of (4.9). But $S(\tau)$ is now well behaved at $\tau=0$; the discontinuity which gives rise to the first term on the right-hand side of (4.7) is replaced by a continuous function with slope proportional to the speed of sound, $c$, say. Compressibility effects persist for a time $a / c$. The viscous relaxation time is of order $M / 4 \pi \mu a$. When $a / c \ll M / 4 \pi \mu a$, as we are supposing to be the case, compressibility effects are unimportant and the correct answer is obtained by using the incompressible equations of motion and including the hydrodynamic mass of the particle. This has been verified in detail by Zwanzig \& Bixon (1975). 


\section{The mean-square displacement of a particle in a sheet}

The velocity field is two-dimensional, in the plane of the sheet. The convective acceleration terms are neglected. Thus, we wish to solve

with

$$
\rho \partial \mathbf{u} / \partial t=-\nabla p+\mu \nabla^{\mathbf{2}} \mathbf{u}, \quad \operatorname{div} \mathbf{u}=0
$$

$$
u_{r}=U(t) \cos \theta, \quad u_{\theta}=-U(t) \sin \theta \quad \text { on } \quad r=a,
$$

and $\mathbf{u} \rightarrow 0$ as $r \rightarrow \infty$. We take Laplace transforms and introduce a stream function $\hat{\psi}(r, \theta, s)$, which satisfies

$$
\begin{gathered}
\rho s \nabla^{2} \hat{\psi}=\mu \nabla^{4} \hat{\psi}, \\
\hat{\psi}(a, \theta, s)=a \hat{U} \sin \theta, \quad \partial \hat{\psi}(a, \theta, s) / \partial r=a \hat{U} \sin \theta .
\end{gathered}
$$

Solution by separation of variables is straightforward, and after some algebra we find that $\hat{F}(s)$, the Laplace transform of the drag $F(t)$, is given by

$$
\hat{F}(s)=\pi \rho a^{2} h s \hat{U}+4 \pi \mu h \xi \frac{K_{1}(\xi)}{K_{0}(\xi)} \hat{U},
$$

where $\xi=\left(\rho a^{2} s / \mu\right)^{\frac{1}{2}}$. Comparing with (4.7), we see that

$$
M^{\prime}=\pi \rho a^{2} h, \quad \hat{G}(s)=4 \pi \mu h \xi K_{1}(\xi) / K_{0}(\xi) .
$$

Here $K_{0}$ and $K_{1}$ denote modified Bessel functions of the second kind, and as $\xi \rightarrow 0$,

$$
\xi \frac{K_{1}(\xi)}{K_{0}(\xi)} \sim \frac{1+\frac{1}{2} \xi^{2}\left(\log \xi-\frac{1}{4}+\frac{1}{2} \gamma\right)+\ldots}{-\left(\log \frac{1}{2} \xi+\gamma\right)+\frac{1}{4} \xi^{2}\left(-\log \frac{1}{2} \xi-\gamma+1\right)+\ldots} .
$$

Note that $\tilde{G}(0)=0$, which means that the mobility is infinite.

To find the asymptotic behaviour of $\left\langle X^{2}(t)\right\rangle$ for large $t$, we use the fact that for small $s$

$$
\left[s+\frac{\widehat{G}(s)}{\left(M+M^{\prime}\right)}\right]^{-1}=\frac{\left(M+M^{\prime}\right)}{4 \pi \mu h}\left[-\frac{1}{2} \log \frac{\rho a^{2} s}{4 \mu}-\gamma+O\left(s \log ^{2} s\right)\right] .
$$

Hence, from tables or by direct evaluation,

$$
\left\langle X^{2}(t)\right\rangle \sim \frac{2 k_{B} T t}{4 \pi \mu h}\left[\log \left(\frac{4 \mu t}{\rho a^{2}}\right)-(1+\gamma)+O\left(\frac{\rho a^{2} \log ^{2} t}{\mu t}\right)\right] .
$$

Notice that now the mean-square displacement grows like $t \log t$, corresponding to a non-constant diffusivity.

The law (5.8) would be relevant, instead of that deduced from (3.10), if $\mu t / \rho a^{2} \ll h \mu / a \mu^{\prime}$. However, $a$ is usually so small for biological applications $\left(a \sim 10^{-7} \mathrm{~cm}\right)$ that this condition is not satisfied in practice, so the results of this section are primarily of academic interest.

\section{The no-slip boundary condition}

To calculate the drag on a molecule by continuum hydrodynamics, with the molecule replaced by a rigid particle whose size is given by the molecular dimensions, is to make a non-trivial assumption. For small molecules, it has been 
noted that the results are sometimes not too good, and it has been suggested that a more appropriate model is to drop the no-slip boundary condition valid for solid bodies in a fluid and replace it by a condition of zero tangential stress (nostick); see Hu \& Zwanzig (1974) and Bauer, Brauman \& Pecora (1974). For the translational diffusion, this makes little difference [see (3.10)]. However, the rotational mobility is now infinite if the particle is a circular cylinder, and a finite mobility requires the assumption of a non-circular shape. For consistency, the translational diffusion should then be recalculated for the same shape.

It is possible to calculate the mobilities of particles of elliptical cross-section (although numerical work seems to be required). But this calculation seems premature at the present stage and has therefore not been completed. Apart from the uncertainty in identifying the molecular dimensions with the size of the equivalent rigid particle, large molecules should presumably be modelled by particles with rough surfaces, and as Richardson (1973) has shown, the no-slip and zero-tangential-stress boundary conditions are then practically equivalent as far as the drag is concerned.

This work was supported by the National Science Foundation, Grant ENG 74-00124.

\section{REFERENCES}

Abramowitz, M. \& Stegun, I. A. 1965 Handbook of Mathematical Functions. Dover. Bauer, D. R., Brauman, J. I. \& Pecora, R. 1974 J. Am. Chem. Soc. 96, 6840.

Edidin, M. 1974 Ann. Rev. Biophys. Bioengng, 3, 179.

Enstein, A. 1956 Theory of Brownian Motion. Dover.

Hu, C. \& Zwanzrg, R. 1974 J. Chem. Phys. 60, 4354.

KUво, R. 1957 J. Phys. Soc. Japan, 12, 570.

LAMB, H. 1932 Hydrodynamics. Cambridge University Press.

Landat, L. D. \& Lirshitz, E. M. 1959 Fluid Mechanics. Pergamon.

Richandson, S. 1973 J. Fluid Mech. 59, 707.

Satfrman, P. G. \& Delbrück, M. 1975 Proc. Nat. Acad. Sci. 72, 3111.

Titchmarsh, E. C. 1948 Theory of Fourier Integrals. Oxford University Press.

Tranter, C. J. 1966 Integral Transforms. Methuen.

Wax, N. (ed.) 1954 Selected Papers on Noise and Stochastic Processes. Dover.

Zwanzia, R. \& Bixon, M. 1975 J. Fluid Mech. 69, 21-25 